\title{
A Re-Evaluation of the Run Rules Control Charts for Monitoring the Coefficient of Variation
}

\author{
Khai Wah Khaw ${ }^{1}$, XinYing Chew ${ }^{2, *}$ \\ ${ }^{1}$ School of Management, Universiti Sains Malaysia, 11800 Pulau Pinang, Malaysia \\ ${ }^{2}$ School of Computer Sciences, Universiti Sains Malaysia, 11800 Pulau Pinang, Malaysia
}

\begin{abstract}
An efficient process monitoring system is important for achieving sustainable manufacturing. The control charting technique is one of the most effective techniques to monitor process quality. In certain processes where the process mean and variance are not independent of one another, the coefficient of variation (CV), which measures the ratio of the standard deviation to the mean should be monitored. Castagliola et al. [21] proposed the two-sided run rules (RR) control charts for monitoring the $\mathrm{CV}$ and it is found that the RR CV charts revealed the problem of ARL-biased performances, especially when the monitored sample size is small, for detecting downward CV shifts. This paper alters the RR CV chart by suggesting the two one-sided run rules (ORR) CV charts achieve the unbiased ARL performances. Additionally, this paper also investigates the ORR CV charts in terms of the expected average run length (EARL) criterion, which is not discussed in [21]. A Markov chain model is established for designing the proposed charts. The statistical performances of the ORR CV, RR CV and Shewhart CV (SH CV) charts are compared in terms of the average run length (ARL) and EARL criteria. The results show that the proposed charts surpass the RR CV and SH CV charts for detecting small and moderate upward and downward CV shifts. The implementation of the ORR CV charts is illustrated with an example using a real dataset.
\end{abstract}

Keywords Average run length, Coefficient of variation, Expected average run length, Markov chain, One-sided run rules.

AMS 2010 subject classifications 90B25, 62P30.

DOI: $10.19139 /$ soic-2310-5070-717

\section{Introduction}

Statistical Process Control (SPC) is a powerful collection of problem-solving tools that can be used to control and improve the quality of a process through the reduction of variability. The importance of the SPC has been shown through a wide variety of research publications, such as those by [28], [17], [1] and [32]. A control chart is one of the effective tools in SPC, it has been used with the considerable amount of success in the industry for monitoring the quality of a production process. Over the years, the implementations of control chart were recently extended to many fields, i.e. chemical, finance, healthcare and many others, where the process mean and variance may not vary independently of each other. This caused the use of the traditional control chart may lead to an erroneous conclusion. To circumvent this problem, it is natural to explore the use of the coefficient of variation (CV), which monitors the ratio of the standard deviation to the mean, in process monitoring. The $\mathrm{CV}$ has been applied to various scientific areas. For example, Creticos et al. [25] applied the CV in dose-response studies. Bedeian and Mossholder [3] used the CV in measuring the diversity whereas Pyne et al. [7] employed the CV to investigate the variability of the competitive performance of Olympic swimmers. More recently, Ye et al. [12] explained the use of CV in detecting the presence of chatter, which is several form of self-excited vibration in a machining process.

*Correspondence to: XinYing Chew (Email: xinying@usm.my). School of Computer Sciences, Universiti Sains Malaysia, 11800 Pulau Pinang, Malaysia.

ISSN 2310-5070 (online) ISSN 2311-004X (print)

Copyright (C) 2019 International Academic Press 
Numerous research works on CV control charts have been made over the years. As pioneers in this field, Kang et al. [6] introduced a Shewhart control chart (SH CV) to monitor the CV, which is very efficient in the detection of large process CV shifts. Later, many intermediate and advanced type of the CV charts have been proposed to improve the sensitivity in detecting small and moderate CV shifts, such as [23], [20], [21], [10], [18] and [4]. According to Tran [16], although exponentially weighted moving average (EWMA) and cumulative sum (CUSUM) type control charts can efficiently detect small and moderate process shifts, these charts are not widely accepted by quality practitioners due to their relative complexity during implementation. Therefore, in some of the real-life scenarios, a quality practitioner prefers to use an intermediate type control chart to monitor the production process due to its simplicity. To fill this gap in research, Castagliola et al. [21] proposed the intermediate control charts to monitor the $\mathrm{CV}$, i.e. the two-sided run rules $\mathrm{CV}$ (RR CV) charts.

Meanwhile, the research works on run rules control charts are still being continually explored by many researchers. Kritzinger et al. [24] suggested the improved run rules nonparametric sign charts. Majika et al. [11] added the run rules schemes into the distribution-free Phase-II Mann-Whitney control charts. The implementation of the run rules charts to monitor the ratio of two normal variables are recommended by [14]. Zhang et al. [31] presented the run rules control charts for monitoring pre-specified changes in linear profiles. Rakitzis [2] evaluated the performance of modified run rules $\bar{x}$ charts with estimated parameters while $\mathrm{Hu}$ and Castagliola [30] studied a run rules $\bar{x}$ chart when the process parameters are unknown. Tran [15] and Tran [13] presented the run rules median and t charts, respectively. Very recently, the run length properties of the synthetic and run rules $\bar{x}$ charts have been explained in zero-state [26] and steady-state [27] schemes.

The two-sided RR CV charts have better statistical performances compared to the SH CV chart in detecting small and moderate CV shifts, in terms of average run length (ARL) and standard deviation run length (SDRL) criteria. However, the two-sided RR CV charts revealed the problem of ARL-biased performances, especially when the monitored sample size is small, for detecting downward CV shifts [21]. Additionally, Acosta-Meija [5] indicated that a single two-sided control chart may produce ARL-biased performances, which may seriously affect the simultaneous monitoring of upward and downward process shifts. The motivation of this research is to overcome the aforementioned problem by proposing two one-sided RR CV charts (ORR CV), for detecting upward and downward CV shifts. The proposed charts able to provide unbiased ARL performances. However, a setback of this research is the exact shift size must be specified prior to the implementation of this scheme as this is required by the ARL criterion. In most of the industrial scenarios, practitioners do not have knowledge of the exact CV shift size. To tackle this issue, this paper also investigates the ORR CV charts in terms of the expected average run length (EARL) criterion, which is not discussed in [21].

The goal of this paper is to overcome the ARL-biased performance of the RR CV chart [21]. Hence, this paper proposes two one-sided run rules schemes, i.e. the 2-out-of-3 run rules $\left(O R R_{2,3} C V\right)$ and the 3-out-of-4 run rules $\left(O R R_{3,4} C V\right)$ charts, for detecting upward and downward $\mathrm{CV}$ shifts. The remainder of this paper is organized as follows: The next section discusses the basic properties of the SH CV chart. In Section 3, the proposed ORR CV charts are described. The derivation of the formulae to compute the ARL, standard deviation of the run length (SDRL) and EARL based on the Markov-chain approach are discussed. The numerical comparisons among the

ORR CV and existing RR CV and SH CV charts, in terms of the ARL, SDRL and EARL criteria, will be shown in Section 4. The implementation of the ORR CV charts will be illustrated with an example based on a real dataset from the manufacturing sector. In the last section, a summary of the findings and suggestions for future research are provided.

\section{Basic Properties of Sample CV}

Suppose that $\mu$ and $\sigma$ are the mean and standard deviation of a positive random variable, $X$, respectively. Then the $\mathrm{CV}$ of $X$ is

$$
\gamma=\frac{\sigma}{\mu}
$$


We assume that $X_{1}, X_{2}, \ldots, X_{n}$ is a random sample of size $\mathrm{n}$ from the normal distribution. Then, $\bar{x}$ and $S$ are the sample mean and sample standard deviation, respectively, given as follows:

$$
\bar{x}=\frac{1}{n} \sum_{i=1}^{n} X_{i}
$$

and

$$
S=\sqrt{\frac{1}{n-1} \sum_{i=1}^{n}\left(X_{i}-\bar{x}\right)^{2}}
$$

The sample $\mathrm{CV}$ is computed as

$$
\hat{\gamma}=\frac{S}{\bar{x}}
$$

For the probability distribution of sample $\mathrm{CV}, \frac{\sqrt{n}}{\hat{\gamma}}$ follows a noncentral $t$ distribution with $n-1$ degrees of freedom and noncentrality parameter $\frac{\sqrt{n}}{\gamma}$. Here, if $\gamma(>0)$ is not too large, say $\gamma \in(0,0.5]$, then the cumulative distribution function (cdf) of $\hat{\gamma}$ can be accurately approximated as

$$
F_{\hat{\gamma}}(x \mid n, \gamma)=1-F_{t}\left(\frac{\sqrt{n}}{x} \mid n-1, \frac{\sqrt{n}}{\gamma}\right),
$$

where $F_{t}($.$) refers to the cdf of a noncentral t$ random variable with $n-1$ degrees of freedom and noncentrality parameter $\sqrt{n} / \gamma$. Inverting $F_{\hat{\gamma}}(x \mid n, \gamma)$ gives the inverse cdf of $\hat{\gamma}$ as

$$
F_{\hat{\gamma}}^{-1}(\alpha \mid n, \gamma)=\frac{\sqrt{n}}{F_{\hat{\gamma}}^{-1}\left(1-\alpha \mid n-1, \frac{\sqrt{n}}{\gamma}\right)}
$$

where $F_{\hat{t}}^{-1}($.$) refers to the inverse cdf of the noncentral t$ random variable with $n-1$ degrees of freedom and noncentrality parameter $\frac{\sqrt{n}}{\gamma}$.

The center line of the SH CV chart is set as the in-control CV value, $\gamma_{0}$, while the charts limits are obtained using probability limits. Two separate one-sided SH CV charts: (i) the upward chart consists of the upper control limit, UCL, for detecting an increase in the CV and (ii) the downward chart consists of the lower control limit, LCL, for detecting a decrease in the CV. By setting the Type-I error probability of each of the charts as $\alpha_{0}$, the limits are obtained as

$$
L C L=F_{\hat{\gamma}}^{-1}\left(\alpha_{0} \mid n, \gamma_{0}\right)
$$

and

$$
U C L=F_{\hat{\gamma}}^{-1}\left(1-\alpha_{0} \mid n, \gamma_{0}\right)
$$

Note that $\alpha_{0}=\frac{1}{A R L_{0}}$, where the in-control ARL $\left(A R L_{0}\right)$ value is specified by the user. The upward and downward SH CV charts detect an out-of-control signal when $\hat{\gamma}$ is plotted above UCL or below LCL, respectively. Therefore, the probability that the SH CV charts issue an out-of-control signal are $B=\operatorname{Pr}(\hat{\gamma}>U C L)$ (for the upward SH CV chart) and $B=\operatorname{Pr}(\hat{\gamma}<L C L)$ (for the downward SH CV chart), respectively. The ARL, SDRL and out-of-control $\operatorname{EARL}\left(E A R L_{0}\right)$ of the SH CV charts are computed as

$$
A R L=\frac{1}{B},
$$




$$
S D R L=\frac{\sqrt{1-B}}{B}
$$

and

$$
E A R L_{1}=\int_{\tau_{\min }}^{\tau_{\max }} A R L_{1}\left(L C L, U C L, B, n, \gamma_{0}, \tau\right) f_{\tau}(\tau) d \tau
$$

where $A R L_{1}$ is the out-of-control ARL and the in-control EARL $\left(E A R L_{0}\right)$ is set equal to $A R L_{0}$. Here, $f_{\tau}(\tau)$ defines the probability density function (pdf) of $\tau$, where $\tau$ is assumed to follow a uniform $U\left(\tau_{\min }, \tau_{\max }\right)$ distribution, while $\tau_{\min }$ and $\tau_{\max }$ denote the lower and upper bound for the shift size $\tau$.

\section{The ORR CV Charts}

The run rules scheme monitors a process by looking at the number of samples, out of a certain number of successive samples taken, which falls outside the warning limits. For instance, a $m$-out-of- $k$ run rules will generate an out-ofcontrol signal if out of $k$ consecutive samples taken, $m$ samples fall outside the warning limits. Incorporating run rules is known to improve the performance of the chart ([9], [8]). This paper proposes $O R R_{2,3} C V$ and $O R R_{3,4} C V$ charts.

\subsection{The Upward and Downward $O R R_{2,3} C V$ Charts}

In the $O R R_{2,3} C V$ charts, an out-of-control signal is produced after two out of three successive sample CVs are plotted above the upper warning limit (UWL) or below the lower warning limit (LWL). There are no control limits in the $O R R_{2,3} C V$ chart. The proposed charts are simple to implement as they only involve a pair of limits. The UWL of the upward $O R R_{2,3} C V$ chart and the LWL of the downward $O R R_{2,3} C V$ chart can be obtained as follows:

$$
L W L=\mu_{0}(\hat{\gamma})-K \sigma_{0}(\hat{\gamma})
$$

and

$$
U W L=\mu_{0}(\hat{\gamma})+K \sigma_{0}(\hat{\gamma})
$$

where $K$ is the parameters of the warning limits. Note that $\mu_{0}(\hat{\gamma})$ and $\sigma_{0}(\hat{\gamma})$ are the mean and standard deviation of sample CV, respectively, when the process is in-control. The following approximations presented by Reh and Scheffler [29] can be used to compute the limits in Equations (12) and (13):

$$
\mu_{0}(\hat{\gamma}) \approx \gamma_{0}\left(1+\frac{1}{n}\left(\gamma_{0}^{2}-\frac{1}{4}\right)+\frac{1}{n^{2}}\left(3 \gamma_{0}^{4}-\frac{\gamma_{0}^{2}}{4}-\frac{7}{32}\right)+\frac{1}{n^{3}}\left(15 \gamma_{0}^{6}-\frac{3 \gamma_{0}^{4}}{4}-\frac{7 \gamma_{0}^{2}}{32}-\frac{19}{128}\right)\right)
$$

and

$$
\sigma_{0}(\hat{\gamma}) \approx \gamma_{0}\left(\frac{1}{n}\left(\gamma_{0}^{2}+\frac{1}{2}\right)+\frac{1}{n^{2}}\left(8 \gamma_{0}^{4}+\gamma_{0}^{2}+\frac{3}{8}\right)+\frac{1}{n^{3}}\left(69 \gamma_{0}^{6}+\frac{7 \gamma_{0}^{4}}{2}+\frac{3 \gamma_{0}^{2}}{4}+\frac{3}{16}\right)\right)^{\frac{1}{2}}
$$

The Markov-chain approach is adopted to derive the formulae for the ARL, SDRL and EARL In both the upward and downward $O R R_{2,3} C V$ charts, the states of the Markov-chain are defined based on the position of the last two sample CVs plotted on the chart. The following three transient states of the upward chart are defined in the Markov chain model:

State 1 (10): 1st sample above UWL and 2nd sample below UWL; State 2 (01): 1st sample below UWL and 2nd sample above UWL; State 3 (00): two successive samples below UWL. 
Then, three transient states of the downward $\mathrm{ORR}_{2,3} \mathrm{CV}$ chart are

State 1 (00): two successive samples above LWL;

State 2 (01): 1st sample above LWL and 2nd sample below LWL;

State 3 (10): 1st sample below LWL and 2nd sample above LWL.

The third point (i.e. the current ith sample $\mathrm{CV}, \hat{\gamma}_{i}$ ) will decide whether the process is in-control or out-ofcontrol, as it will determine whether two or less than two sample CVs will fall outside the warning limits. The following shows the transition probability matrix (tpm) without absorbing states for both the upward and downward $O R R_{2,3} C V$ charts:

$$
\boldsymbol{U}_{2,3}=\left(\begin{array}{cc}
\boldsymbol{Q}_{2,3} & \boldsymbol{r} \\
\mathbf{0}^{T} & \mathbf{1}
\end{array}\right)=\left(\begin{array}{ccc}
0 & 0 & 1-P_{U} \\
1-P_{U} & 0 & 0 \\
0 & P_{U} & 1-P_{U}
\end{array}\right)
$$

and

$$
\boldsymbol{D}_{2,3}=\left(\begin{array}{cc}
\boldsymbol{Q}_{2,3} & \boldsymbol{r} \\
\mathbf{0}^{T} & \mathbf{1}
\end{array}\right)=\left(\begin{array}{ccc}
1-P_{L} & P_{L} & 0 \\
0 & 0 & 1-P_{L} \\
1-P_{L} & 0 & 0
\end{array}\right)
$$

Note that $\boldsymbol{Q}_{2,3(3 x 3)}$ is the transition probability matrix of transient probabilities while the vector $\boldsymbol{r}$ satisfies $\boldsymbol{r}=\mathbf{1}-\boldsymbol{Q}_{2,3} \mathbf{1}$, i.e. the sum of the row probabilities are equal to unity. Note also that $\mathbf{0}^{T}=(0,0,0)$ and $\mathbf{1}=(1,1,1)^{T}$. The initial probabilities for both the upward and downward $O R R_{2,3} C V$ charts are $\boldsymbol{q}_{2,3}=(0,0,1)^{T}$ and $\boldsymbol{q}_{2,3}=(1,0,0)^{T}$, respectively. $P_{U}$ and $P_{L}$ are computed as

$$
P_{U}=\operatorname{Pr}(\hat{\gamma} \geq U W L)=1-F_{\hat{\gamma}}\left(U W L \mid n, \gamma_{1}\right)
$$

and

$$
P_{L}=\operatorname{Pr}(\hat{\gamma} \leq L W L)=F_{\hat{\gamma}}\left(L W L \mid n, \gamma_{1}\right),
$$

where $F_{\hat{\gamma}}($.$) is the cdf of \hat{\gamma}$ and $\gamma_{1}=\tau \gamma_{0}$. Here, $\tau$ represents the shift size in the CV. The ARL and SDRL for both the upward and downward $O R R_{2,3} C V$ charts can be obtained as

$$
A R L=\boldsymbol{q}_{2,3}^{T}\left(\boldsymbol{I}-\boldsymbol{Q}_{2,3}\right)^{-1} \mathbf{1},
$$

and

$$
S D R L=\sqrt{2 \boldsymbol{q}_{2,3}^{T}\left(\boldsymbol{I}-\boldsymbol{Q}_{2,3}\right)^{-2} \boldsymbol{Q}_{2,3} \mathbf{1}-A R L^{2}+A R L .}
$$

In the computation of the ARL, the shift size, $\tau$ needs to be specified a priori. If $\tau$ could not be specified, the EARL can be used as a performance measure to monitor the CV [20], [19]. In this paper, the $E A R L_{0}$ is set equal to $A R L_{0}$, while the $E A R L_{1}$ is computed as

$$
E A R L_{1}=\int_{\tau_{\min }}^{\tau_{\max }} A R L_{1}\left(L W L(\operatorname{or} U W L), K, n, \gamma_{0}, \tau\right) f_{\tau}(\tau) d \tau
$$

where $f_{\tau}(\tau)$ denotes the pdf of $\tau$. If there is no information on $f_{\tau}(\tau)$, then it is often hard to fit the actual shape of $f_{\tau}(\tau)$. In this scenario, one can assume that $\tau$ follows a uniform distribution over the interval $\left(\tau_{\min }, \tau_{\max }\right)$. Castagliola et al. [23] recommended the interval $\left(\tau_{\min }, \tau_{\max }\right)=[0.5,1)$ for the downward chart and the interval $\left(\tau_{\min }, \tau_{\max }\right)=(1,2]$ for the upward chart. 


\subsection{The Upward and Downward $O R R_{3,4} C V$ Charts}

In the $O R R_{3,4} C V$ charts, they consist of seven in-control states, depending on the position of the last three sample $\mathrm{CV}$ s plotted on the chart. The following seven transient states of the upward $O R R_{3,4} C V$ chart are defined in the Markov chain model:

State 1 (110): 1st and 2nd samples above UWL and 3rd sample below UWL;

State 2 (101): 1st sample above UWL, 2nd sample below UWL and 3rd sample above UWL;

State 3 (100): 1st sample above UWL, 2nd and 3rd samples below UWL;

State 4 (011): 1st sample below UWL, 2nd and 3rd samples above UWL;

State 5 (010): 1st sample below UWL, 2nd sample above UWL, and 3rd sample below UWL;

State 6 (001): 1st and 2nd samples below UWL and 3rd sample above UWL;

State 7 (000): three successive samples below UWL.

Then, three transient states of the downward $O R R_{3,4} C V$ chart are

State 1 (000): three successive samples above LWL;

State 2 (001): 1st and 2nd samples above LWL and 3rd sample below LWL;

State 3 (010): 1st sample above LWL, 2nd sample below LWL and 3rd sample above LWL;

State 4 (011): 1st sample above LWL, 2nd and 3rd samples below LWL;

State 5 (100): 1st sample below LWL, 2nd and 3rd samples above LWL;

State 6 (101): 1st sample below LWL, 2nd sample above LWL and 3rd sample below LWL;

State 7 (110): 1st and 2nd samples below LWL and 3rd sample above LWL.

The location of the current $i$ th sample $\mathrm{CV}, \hat{\gamma}_{i}$, on the chart will decide whether the process is in-control or outof-control, as it will determine whether three or less than three sample CVs will fall outside the warning limits. The $O R R_{3,4} C V$ chart signals an out-of-control if three out of four successive sample CVs plot above UWL (for the upward $O R R_{3,4} C V$ chart) or below LWL (for the downward $O R R_{3,4} C V$ chart). The upper and lower warning limits of the $O R R_{3,4} C V$ chart can be computed using Equations (12) and (13).

Consequently, the ARL, SDRL and EARL of the $O R R_{3,4} C V$ charts are computed using Equations (20), (21) and (22), respectively, by replacing $\boldsymbol{q}_{2,3}^{T}$ and $\boldsymbol{Q}_{2,3}$ with $\boldsymbol{q}_{3,4}^{T}$ and $\boldsymbol{Q}_{3,4}$, respectively. Here, $\boldsymbol{q}_{3,4}=(0,0,0,0,0,0,1)^{T}$ for the upward chart and $\boldsymbol{q}_{3,4}=(1,0,0,0,0,0,0)^{T}$ for the downward chart, while $\boldsymbol{Q}_{3,4}$ is a (7 x 7) tpm. As in the $O R R_{3,4} C V$ charts, the vector $\boldsymbol{r}$ satisfies $\boldsymbol{r}=\mathbf{1}-\boldsymbol{Q}_{3,4} \mathbf{1}$, i.e. the sum of the row probabilities are equal to unity. The following shows the tpm without absorbing states for both the upward and downward $O R R_{3,4} C V$ charts:

$$
\boldsymbol{U}_{3,4}=\left(\begin{array}{cc}
\boldsymbol{Q}_{3,4} & \boldsymbol{r} \\
\mathbf{0}^{T} & \mathbf{1}
\end{array}\right)=\left(\begin{array}{ccccccc}
0 & 0 & 1-P_{U} & 0 & 0 & 0 & 0 \\
0 & 0 & 0 & 0 & 1-P_{U} & 0 & 0 \\
0 & 0 & 0 & 0 & 0 & P_{U} & 1-P_{U} \\
1-P_{U} & 0 & 0 & 0 & 0 & 0 & 0 \\
0 & P_{U} & 1-P_{U} & 0 & 0 & 0 & 0 \\
0 & 0 & 0 & P_{U} & 1-P_{U} & 0 & 0 \\
0 & 0 & 0 & 0 & 0 & P_{U} & 1-P_{U}
\end{array}\right)
$$

and

$$
\boldsymbol{D}_{3,4}=\left(\begin{array}{cc}
\boldsymbol{Q}_{3,4} & \boldsymbol{r} \\
\mathbf{0}^{T} & \mathbf{1}
\end{array}\right)=\left(\begin{array}{ccccccc}
1-P_{L} & P_{L} & 0 & 0 & 0 & 0 & 0 \\
0 & 0 & 1-P_{L} & P_{L} & 0 & 0 & 0 \\
0 & 0 & 0 & 0 & 1-P_{L} & P_{L} & 0 \\
0 & 0 & 0 & 0 & 0 & 0 & 1-P_{L} \\
1-P_{L} & P_{L} & 0 & 0 & 0 & 0 & 0 \\
0 & 0 & 1-P_{L} & 0 & 0 & 0 & 0 \\
0 & 0 & 0 & 0 & 1-P_{L} & 0 & 0
\end{array}\right)
$$




\section{Numerical Comparison}

In this paper, the performance of the proposed charts are evaluated in terms of the unbiased ARL, SDRL and EARL. The $A R L_{0}$ is set as 370.4. Once $K$ is defined based on $A R L_{0}$, the $A R L_{1}$ and $E A R L_{1}$ can be numerically evaluated for a particular shift size $(\tau)$ and for a particular range of shift sizes $\left(\tau_{\min }, \tau_{\max }\right)$, respectively. In this paper, we consider $n \in 5,10,15, \gamma_{0} \in 0.10,0.15,0.20$, for $\tau_{0} \in 0.5,0.6,0.7,0.8,0.9,1.1,1.2,1.5,2.0,2.5$.

Tables 1 and 2 present the $A R L_{1}$ and $S D R L_{1}$ values for the $O R R_{2,3} C V, O R R_{3,4} C V$, $R R_{2,3} C V$ [21], $R R_{3,4} C V$ [21] and $\mathrm{SH} \mathrm{CV}$ [6] charts, for $n \in 5,10,15, \gamma_{0} \in 0.10,0.15,0.20$, for $\tau_{0} \in 0.5,0.6,0.7,0.8,0.9,1.1,1.2,1.5,2.0,2.5$. From Tables 1 and 2 , it is clear that the ORR CV charts prevail over the RR CV and SH CV charts, in terms of the $A R L_{1}$ and $S D R L_{1}$ criteria, for detecting both upward and downward $\mathrm{CV}$ shifts. This means that the proposed charts provide unbiased ARL and SDRL performances. For example, in Table 2, when $\tau=0.9, n=10$, and $\gamma_{0}=0.15, A R L_{1}=82.8,183.7,166.9$ and $S D R L_{1}=80.1,180.9,166.4$, for the $O R R_{3,4} C V, R R_{3,4} C V$ and SH CV charts, respectively, where the $O R R_{3,4} C V$ charts have the smallest $A R L_{1}$ and $S D R L_{1}$ values.

Table 3 shows the performances of the $O R R_{2,3} C V, O R R_{3,4} C V, R R_{2,3} C V$ [21], $R R_{3,4} C V$ [21] and SH CV [6] charts, in terms of the $E A R L_{1}$ criterion, for $n \in 5,10,15, \gamma_{0} \in 0.10,0.15,0.20,\left(\tau_{\min }, \tau_{\max }\right)=[0.5,1),(1,2]$. The ORR CV charts outperform the RR CV and SH CV charts for detecting both upward and downward shifts in the interval $\left(\tau_{\min }, \tau_{\max }\right)=[0.5,1),(1,2]$. For example, when $n=5, \gamma_{0}=0.20$ and $\left(\tau_{\min }, \tau_{\max }\right)=[0.5,1)$, $E A R L_{1}=100.7,82.0,845.9,181.4$ and 149.0, for the $O R R_{2,3} C V, O R R_{3,4} C V, R R_{2,3} C V, R R_{3,4} C V$ and $\mathrm{SH}$ $\mathrm{CV}$ charts, respectively, where the ORR CV charts have the smallest $E A R L_{1}$ value.

\section{An Illustrated Example}

In this section, the implementation of the $O R R_{2,3} C V$ chart is illustrated using a real dataset adopted from Castagliola [22]. The data deal with die casting hot chamber process manufacturing zinc alloy (ZAMAK) parts for the sanitary sector. The Phase-I data contain $m=30$ samples, each with $n=5$ measurements. The sample means, sample standard deviations and sample CVs, for the 30 Phase-I samples are computed and listed in Table 4. The in-control sample $\mathrm{CV}, \hat{\gamma_{0}}$ is computed from the Phase-I data, for $i=1,2, ? 30$, using Equation (4) as

$$
\hat{\gamma_{0}}=\frac{\sum_{i=1}^{30} \hat{\gamma}_{i}}{30}=0.00975 .
$$

By setting the probability of the Type-I error, $\alpha=0.0027$, the lower and upper limits of the downward SH CV and upward SH CV charts are computed using Equations (7) and (8) as

$$
L C L=F_{\hat{\gamma}}^{-1}(0.0027 \mid 5,0.00975)
$$

and

$$
U C L=F_{\hat{\gamma}}^{-1}(1-0.0027 \mid 5,0.00975)
$$

respectively. The upward and downward SH CV charts are plotted in Figures (1) and (2), respectively. Figures (1) and (2) show that $\hat{\gamma}_{i}$ are plotted based on the 30 Phase-I sample CVs, $\hat{\gamma}_{i}$ (for $i=1,2, ? 30$ ). As all the $\hat{\gamma}_{i}$ are plotted below the UCL and above the LCL, the Phase-I process is in-control.

From the Phase-I analysis, $\hat{\gamma_{0}}=0.00975$. In the Phase-II analysis, we assume that $n=5$ and $A R L_{0}=370.4$ are considered. The Phase-II data presented in Table 4 are used to construct the upward and downward $O R R_{2,3} C V$ charts, where the charts are designed to compute the $A R L_{1}$ for upward CV shift size, $\tau=1.2$ and downward $\mathrm{CV}$ shift size, $\tau=0.8$. The parameter $\mathrm{K}$ is obtained as 1.9058 and 1.6065 using nonlinear equation solver for the upward and downward $O R R_{2,3} C V$ charts, respectively. Then, $\mu_{0}(\hat{\gamma})=0.0092$ and $\sigma_{0}(\hat{\gamma})=0.0033$ can be computed from Equations (12) and (13). Subsequently, the warning limits are computed as

$$
L W L=0.0092-1.6065(0.0033)=0.0038
$$




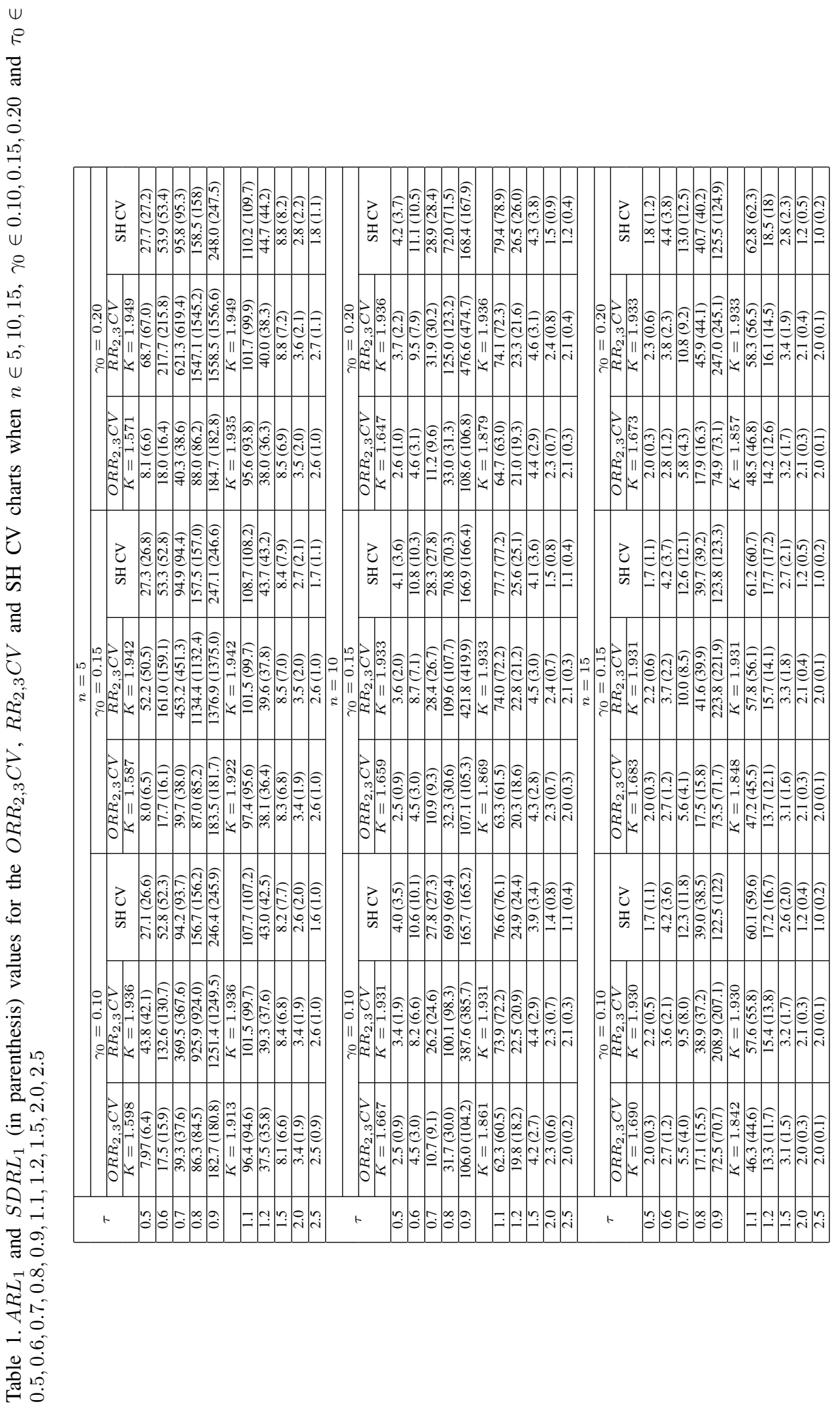




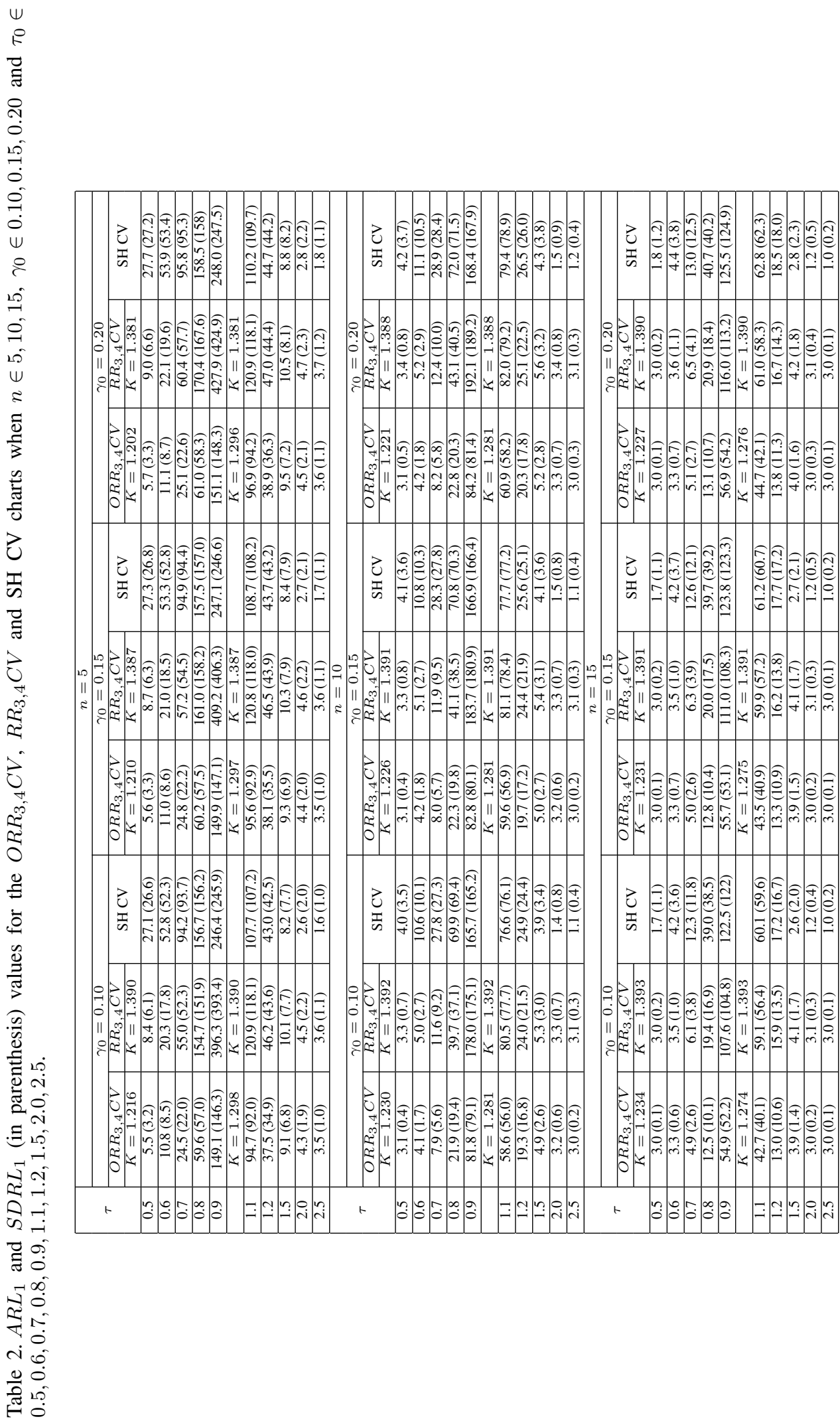




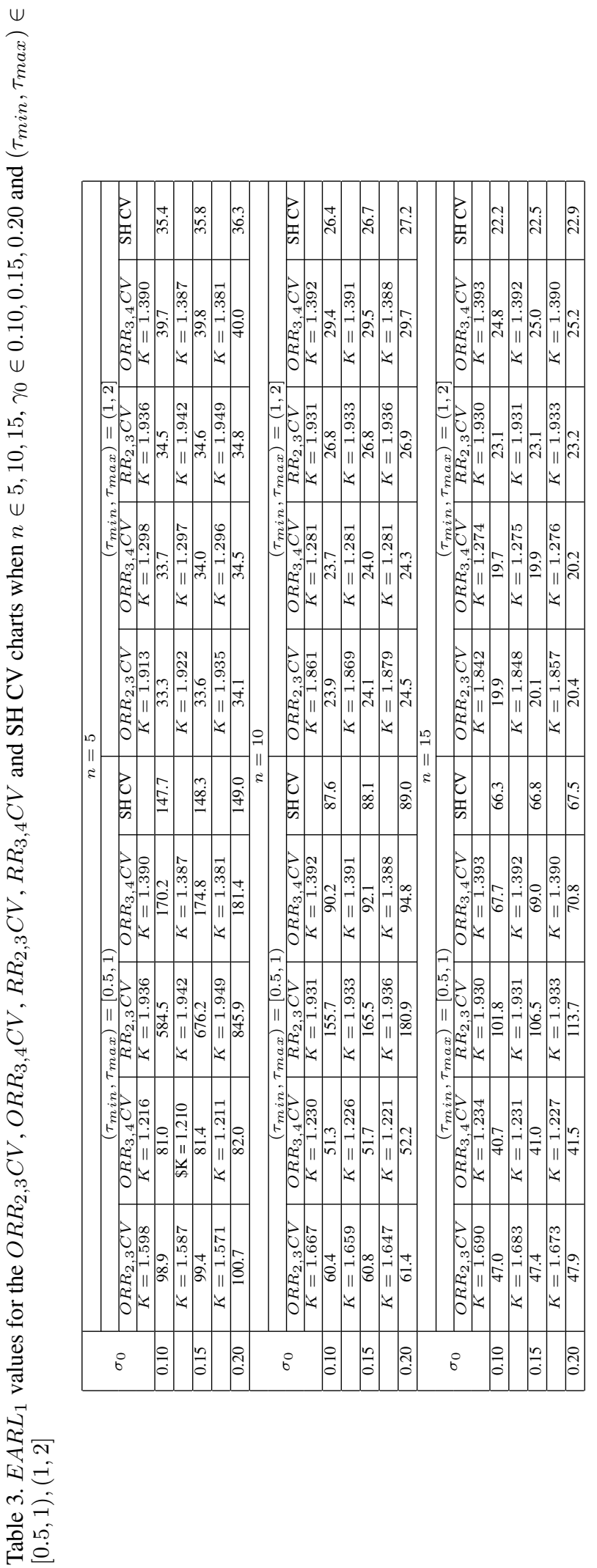

Stat., Optim. Inf. Comput. Vol. 7, December 2019 
Table 4. Phase-I and Phase-II dataset

\begin{tabular}{|c|c|c|c|c|c|c|c|}
\hline \multicolumn{4}{|c|}{ Phase-I } & \multicolumn{4}{|c|}{ Phase-II } \\
\hline$i$ & $\bar{x}_{i}$ & $S_{i}$ & $\hat{\gamma}_{i}$ & $i$ & $\bar{x}_{i}$ & $S_{i}$ & $\hat{\gamma}_{i}$ \\
\hline 1 & 292.6 & 2.701 & 0.0092 & 1 & 396.4 & 4.037 & 0.0102 \\
\hline 2 & 289.0 & 0.707 & 0.0024 & 2 & 393.2 & 1.923 & 0.0049 \\
\hline 3 & 291.4 & 2.073 & 0.0071 & 3 & 404.6 & 3.049 & 0.0075 \\
\hline 4 & 288.0 & 3.937 & 0.0137 & 4 & 396.0 & 2.449 & 0.0062 \\
\hline 5 & 290.0 & 0.707 & 0.0024 & 5 & 301.4 & 3.049 & 0.0101 \\
\hline 6 & 288.2 & 1.303 & 0.0045 & 6 & 295.4 & 1.816 & 0.0061 \\
\hline 7 & 535.4 & 8.264 & 0.0154 & 7 & 293.2 & 1.788 & 0.0061 \\
\hline 8 & 518.4 & 7.224 & 0.0139 & 8 & 297.4 & 2.190 & 0.0074 \\
\hline 9 & 529.2 & 9.203 & 0.0174 & 9 & 642.8 & 2.280 & 0.0035 \\
\hline 10 & 527.0 & 9.591 & 0.0182 & 10 & 640.2 & 1.095 & $\mathbf{0 . 0 0 1 7}$ \\
\hline 11 & 533.6 & 4.929 & 0.0092 & 11 & 650.4 & 3.435 & 0.0053 \\
\hline 12 & 439.2 & 3.114 & 0.0071 & 12 & 647.8 & 1.643 & 0.0025 \\
\hline 13 & 447.2 & 2.774 & 0.0062 & 13 & 646.0 & 2.345 & $\mathbf{0 . 0 0 3 6}$ \\
\hline 14 & 443.4 & 8.173 & 0.0184 & 14 & 549.8 & 3.114 & 0.0057 \\
\hline 15 & 434.0 & 2.549 & 0.0059 & 15 & 522.6 & 10.310 & 0.0197 \\
\hline 16 & 436.0 & 1.224 & 0.0028 & 16 & 519.8 & 7.259 & 0.0140 \\
\hline 17 & 437.6 & 2.408 & 0.0055 & 17 & 518.8 & 8.927 & 0.0172 \\
\hline 18 & 419.6 & 4.307 & 0.0096 & 18 & 515.4 & 11.760 & $\mathbf{0 . 0 2 2 8}$ \\
\hline 19 & 422.4 & 4.159 & 0.0098 & 19 & 550.4 & 15.678 & 0.0285 \\
\hline 20 & 416.8 & 3.962 & 0.0095 & 20 & 529.0 & 10.440 & $\mathbf{0 . 0 1 9 7}$ \\
\hline 21 & 420.4 & 4.979 & 0.0118 & 21 & 526.8 & 9.602 & 0.0182 \\
\hline 22 & 421.6 & 2.302 & 0.0055 & 22 & 529.2 & 7.949 & 0.0150 \\
\hline 23 & 418.4 & 4.393 & 0.0105 & 23 & 521.8 & 7.981 & 0.0153 \\
\hline 24 & 410.4 & 4.219 & 0.0103 & 24 & 534.0 & 7.681 & 0.0144 \\
\hline 25 & 449.0 & 6.204 & 0.0138 & 25 & 525.0 & 5.656 & 0.0108 \\
\hline 26 & 441.6 & 3.781 & 0.0086 & 26 & 533.0 & 5.522 & 0.0104 \\
\hline 27 & 393.2 & 6.220 & 0.0158 & 27 & 287.8 & 3.114 & 0.0108 \\
\hline 28 & 401.8 & 1.483 & 0.0037 & 28 & 287.2 & 3.271 & 0.0114 \\
\hline 29 & 412.6 & 3.049 & 0.0074 & 29 & 289.8 & 1.095 & 0.0038 \\
\hline 30 & 461.4 & 7.700 & 0.0167 & 30 & 288.4 & 3.049 & 0.0106 \\
\hline
\end{tabular}

and

$$
U W L=0.0092+1.9058(0.0033)=0.0155
$$

The upward and downward $O R R_{2,3} C V$ charts are plotted in Figures (3) and (4), respectively, based on the $\hat{\gamma}_{i}$ values in Phase-II data. Figure (3) shows that the downward $O R R_{2,3} C V$ chart detects two out-of-control samples, i.e. at the 10th and 13th samples whereas Figure (4) presents the upward $O R R_{2,3} C V$ chart detects two out-of-control signals at 18th and 20th samples (see the boldfaced values in Table 4). Following these out-of-control samples, the practitioner should investigate the underlying process to find the assignable cause(s) present and take the necessary corrective actions so that the process returns to the in-control situation again. 


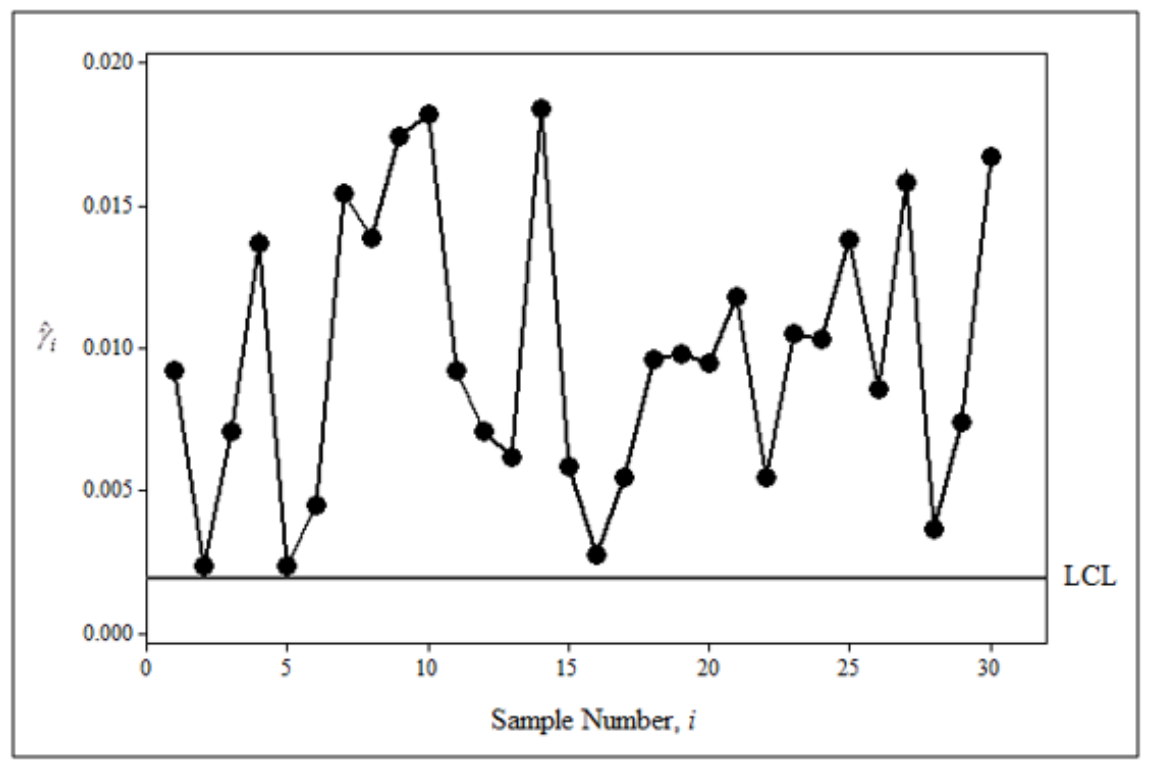

Figure 1. Downward SH CV chart for the Phase-I data

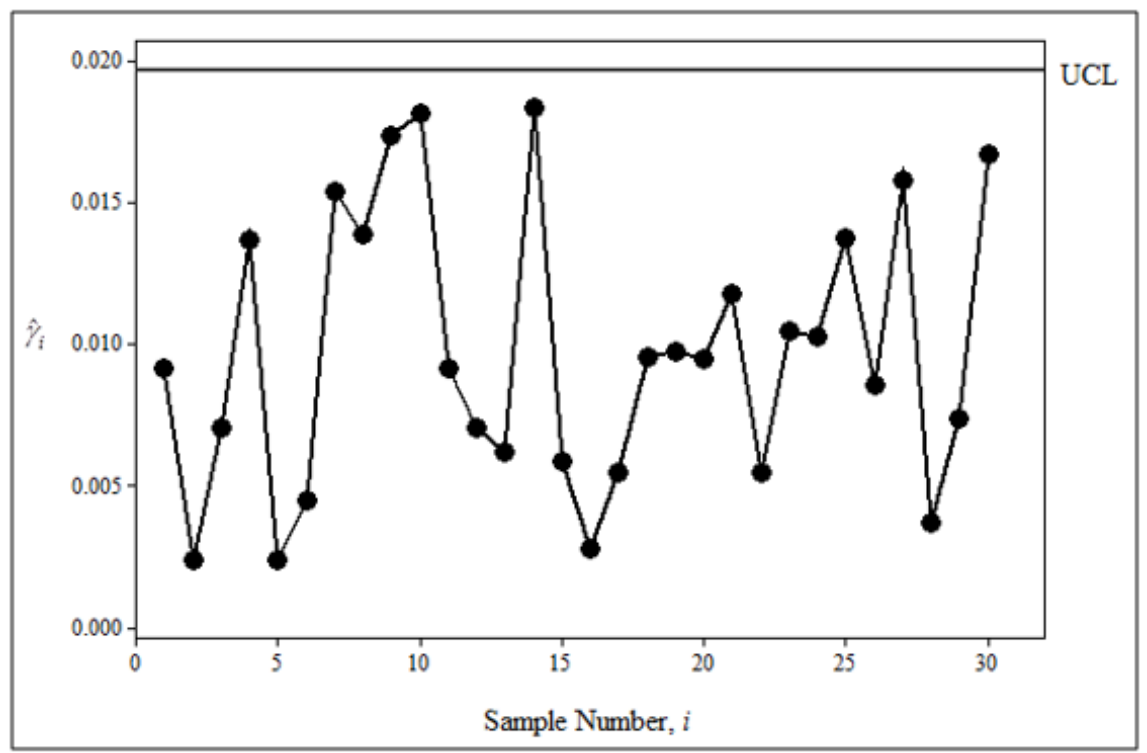

Figure 2. Upward SH CV chart for the Phase-I data

\section{Conclusion}

This paper proposes one-sided run rules control charts for monitoring the CV (ORR CV) to circumvent the ARLbiased performances of the two-sided RR CV charts presented by Castagliola [21]. Additionally, this paper also considers the EARL as a performance measure to evaluate the proposed charts, which is very useful when the $\mathrm{CV}$ shifts could not be specified. The computation of the $A R L_{1}, S D R L_{1}$ and $E A R L_{1}$ are enumerated in this paper. From the numerical comparison, the proposed upward and downward $O R R_{2,3} C V$ and $O R R_{3,4} C V$ charts provide unbiased ARL and EARL performances and they outperform the existing RR CV and SH CV charts in detecting 


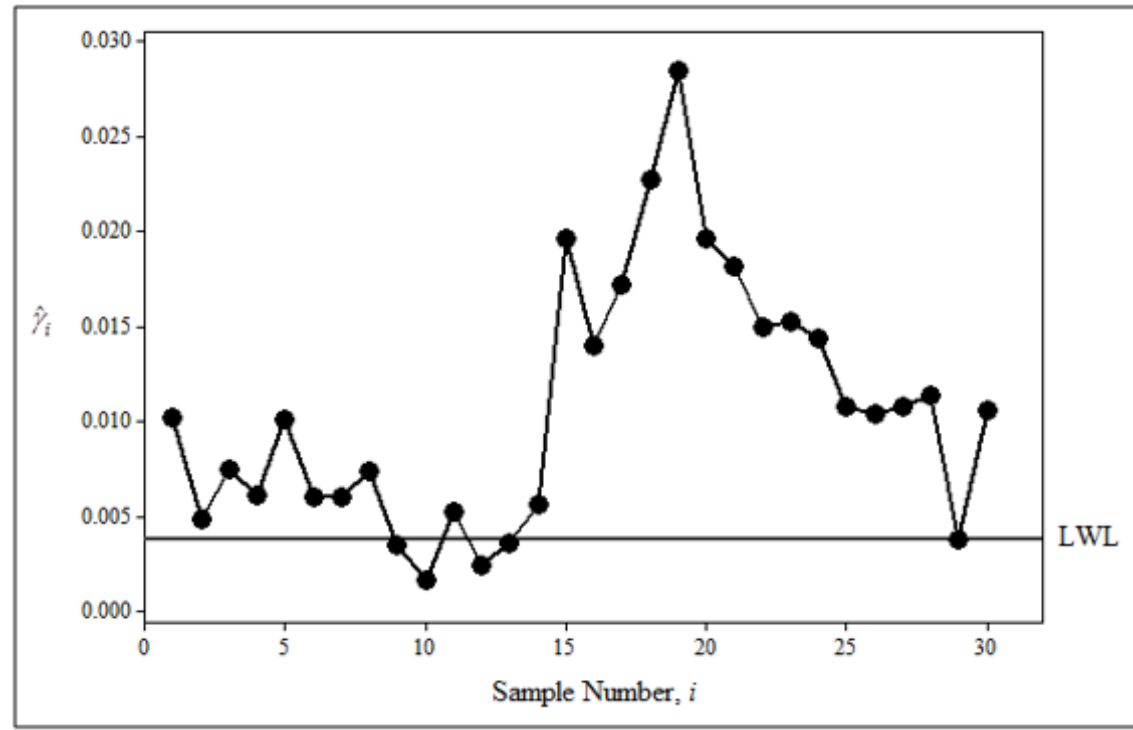

Figure 3. Downward $O R R_{2,3} C V$ chart for the Phase-II data

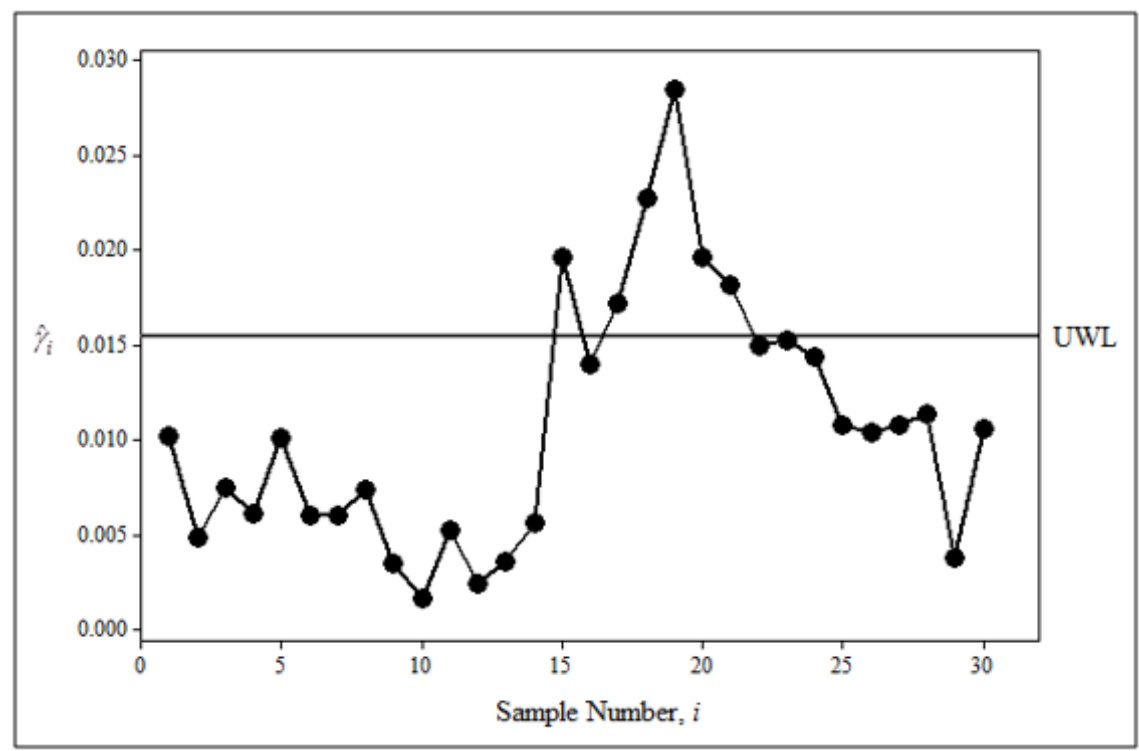

Figure 4. Downward $O R_{2,3} C V$ chart for the Phase-II data

small and moderate $\mathrm{CV}$ shifts. The implementation of the upward and downward $O R R_{2,3} C V$ charts are shown using a real set of manufacturing data. This research can be considered as a framework for quality practitioners who preferred to implement an intermediate type of control chart. The computational difficulty of the ORR charts for monitoring the $\mathrm{CV}$ is similar to that of the traditional SH CV chart, but the ORR CV charts provide better performance. By applying the proposed charts, the quality practitioners can perform quality monitoring easily and efficiently. At the same time, they are able to detect an out-of-control signal in a shorter time. This shows that the proposed charts should be implemented in practical applications. As future research, it is suggested to study the $\mathrm{CV}$ charts based on estimated parameters when the in-control parameters are unknown. 


\section{Acknowledgement}

This work was supported by the Universiti Sains Malaysia, Short Term Grant [Grant Number: 304/PKOMP/6315170].

\section{REFERENCES}

1. A. Bourega, R. Sahraoui, Optimal control of the minimal time crisis problem by non-smooth analysis, Statistics, Optimization and Information Computing, vol. 7, pp. 417-438, 2019.

2. A. C. Rakitzis, On the performance of modified runs rules $\bar{x}$ charts with estimated parameters, Communications in StatisticsSimulation and Computation, vol. 46, pp. 1360-1380, 2017.

3. A. G. Bedeian, K. W. Mossholder, On the use of the coefficient of variation as a measure of diversity, Organizational Research Methods, vol. 3, pp. 285-297, 2000.

4. A. N. B. Muhammad, W. C. Yeong, Z. L. Chong, S. L. Lim, M. B. C. Khoo, Monitoring the coefficient of variation using a variable sample size EWMA chart, Computers \& Industrial Engineering, vol. 126, pp. 378-398, 2018

5. C. A. Acosta-Meija, Monitoring reduction in variability with the range, IIE Transactions, vol. 30, pp. 515-523, 1998.

6. C. W. Kang, M. S. Lee, Y. J. Seong, D. M. Hawkins, A control chart for the coefficient of variation, Journal of Quality Technology, vol. 39, pp. 151-158, 2007.

7. D. B. Pyne, C. B. Trewin, W. G. Hopkins, Progression and variability of competitive performance of Olympic swimmers, Journal of Sports Sciences, vol. 22, pp. 613-620, 2004.

8. D. K. Shepherd, S. E. Rigdon, C. W. Champ, Using runs rules to monitor an attribute chart for Markov process, Quality Technology \& Quantitative Management, vol. 9, pp. 383-406, 2012.

9. D. L. Antzoulakos, A. C. Rakitzis, Runs rules schemes for monitoring process variability, Journal of Applied Statistics, vol. 37, pp. 1231-1247, 2010.

10. H. W. You, M. B. C. Khoo, P. Castagliola, A. Haq, Monitoring the coefficient of variation using the side sensitive group runs chart, Quality and Reliability Engineering International, vol. 32, pp. 1913-927, 2016.

11. J. C. M. Majika, S. Chakraborti, M. A. Graham, Distribution-free Phase II Mann-Whitney control charts with runs-rules, The International Journal of Advanced Manufacturing Technology, vol. 86, pp. 723-735, 2016.

12. J. Ye, P. Feng, C. Xu, Y. Ma, S. Huang, A novel approach for chatter online monitoring using coefficient of variation in machining process, The International Journal of Advanced Manufacturing Technology, vol. 96, pp. 287-297, 2018.

13. K. P. Tran, Designing of run rules $t$ control charts for monitoring changes in the process mean, Chemometrics and Intelligent Laboratory Systems, vol. 174, pp. 85-93, 2018

14. K. P. Tran, P. Castagliola, G. Celano, Monitoring the ratio of two normal variables using run rules type control charts, International Journal of Production Research, vol. 54, pp. 1670-1688, 2016.

15. K. P. Tran, Run rules median control charts for monitoring process mean in manufacturing, Quality and Reliability Engineering International, vol. 33, pp. 2437-2450, 2017.

16. K. P. Tran, The efficiency of the 4-out-of-5 runs rules scheme for monitoring the ratio of population means of a bivariate normal distribution International Journal of Reliability, Quality and Safety Engineering, vol. 23, pp. 1?6, 2016.

17. K. W. Khaw, M. B. C. Khoo, P. Castagliola, M. A. Rahim, New adaptive control charts for monitoring the multivariate coefficient of variation, Computers \& Industrial Engineering, vol. 126, pp. 595-610, 2018.

18. K. W. Khaw, M. B. C. Khoo, W. C. Yeong, Z. Wu, Monitoring the coefficient of variation using a variable sample size and sampling interval control chart, Communications in Statistics - Simulation and Computation, vol. 46, pp. 5772-5794, 2017.

19. K. W. Khaw, X. Y. Chew, W. C. Yeong, W.C. S. L. Lim, Optimal design of the synthetic control chart for monitoring the multivariate coefficient of variation, Chemometrics and Intelligent Laboratory System, vol. 186, pp. 33-40, 2019.

20. M. Calzada, S. Scariano, A synthetic control chart for the coefficient of variation, Journal of Statistical Computation and Simulation, vol. 83, pp. 853-867, 2013.

21. P. Castagliola, A. Achouri, H. Taleb, G. Celano, S. Psarakis, Monitoring the coefficient of variation using control charts with runs rules, Quality Technology \& Quantitative Management, vol. 10, pp. 75-94, 2013.

22. P. Castagliola, A. Achouri, H. Taleb, G. Celano, S. Psarakis, Monitoring the coefficient of variation using a variable sampling interval control chart, Quality and Reliability Engineering International, vol. 29, pp. 1135-1149, 2013.

23. P. Castagliola, G. Celano, S. Psarakis, Monitoring the coefficient of variation using EWMA charts, Journal of Quality Technology, vol. 43, pp. 249-265, 2011.

24. P. Kritzinger, S. W. Human, S. Chakraborti, Improved Shewhart-type runs rules nonparametric sign charts, Communications in Statistics ?Theory and Methods, vol. 43, pp. 4723-4748, 2014.

25. P. S. Creticos, W. R. Adams, B. G. Petty, A methacholine challenge dose-response study for development of a pharmaco dynamic bioequivalence methodology for albuterol metered-dose inhalers, Journal of Allergy Clinical Immunization, vol. 100, pp. 713-720, 2002.

26. S. C. Shongwe, M. A. Graham, Some theoretical comments regarding the run length properties of the synthetic and runs-rules $\bar{x}$ monitoring schemes ?part 1: zero-state, Quality Technology \& Quantitative Management, vol. 16, pp. 170-189, 2019.

27. S. C. Shongwe, M. A. Graham, Some theoretical comments regarding the run length properties of the synthetic and runs-rules $\bar{x}$ monitoring schemes ?part 2: steady-state, Quality Technology \& Quantitative Management, vol. 16, pp. 190-199, 2019.

28. S. Noorian, M. N. Ahmadabadi, The use of the extended generalized lambda distribution for controlling the statistical process in individual measurements, Statistics, Optimization and Information Computing, vol. 6, pp. 536-546, 2018. 
29. W. Reh, B. Scheffler, Significant tests and confidence intervals for coefficients of variation, Computational Statistics \& Data Analysis vol. 22, pp. 449-452, 1996.

30. X. L. Hu, P. Castagliola, A re-evaluation of the run rules $\bar{x}$ chart when the process parameters are unknown, Quality Technology $\&$ Quantitative Management, in press. Doi: https://doi.org/10.1080/16843703.2018.1513826

31. Y. Zhang, Y. Shang, N. Gao, Q. Wang, Monitoring the prespecified changes in linear profiles using control charts with supplementary runs rules, Communications in Statistics ?Simulation and Computation, vol. 46, pp. 7249-7263, 2017.

32. Z. L. Chong, M. B. C. Khoo, W. L. Teoh, H. W. You, P. Castagliola, Optimal design of the side-sensitive modified group runs (SSMGR) $\bar{x}$ chart when process parameters are estimated, Quality and Reliability Engineering International, vol. 35, pp. 246-262, 2019. 